\title{
Relation of the equatorial component of the cosmic ray anisotropy to the parameters of interplanetary medium
}

\author{
Abunina Maria \\ Pushkov Institute of Terrestrial Magnetism, Ionosphere and Radio wave propagation (IZMIRAN) \\ Kaluzhskoe Hwy 4, Troitsk, Moscow, 142190, Russia \\ E-mail: abuninaeizmiran.ru

\section{Abunin Artem, Belov Anatoly, Eroshenko Evgeniya, Oleneva Victoria, Yanke Victor} \\ Pushkov Institute of Terrestrial Magnetism, Ionosphere and Radio wave propagation (IZMIRAN) \\ Kaluzhskoe Hwy 4, Troitsk, Moscow, 142190, Russia \\ E-mails: abunineizmiran.ru, abeloveizmiran.ru, erosh@izmiran.ru, \\ olene@izmiran.ru, yanke@izmiran.ru
}

\section{Kryakunova Olga ${ }^{1}$}

Institute of Ionosphere

Kamenskoe Plato, Almaty, Kazakhstan

E-mail: krolganikeyandex.kz

\begin{abstract}
Variations of the cosmic ray vector anisotropy observed on Earth are closely related on the condition of near Earth interplanetary medium. The hourly characteristics of vector anisotropy obtained by the global survey method from the data of world wide neutron monitor network during 1957-2013 allow us to investigate connection of the cosmic ray anisotropy with the solar wind parameters. In the offered work relation of the equatorial component Axy of the cosmic ray anisotropy (for $10 \mathrm{GV}$ rigidity) to the solar wind velocity and density, to intensity of the interplanetary magnetic field and to the changes of cosmic ray density in which the spatial gradient of CR is revealed in interplanetary space, is studied. Characteristics of CR anisotropy for various combinations of the interplanetary parameters corresponding to various conditions of the interplanetary medium are compared. Opportunity to judge on condition of a solar wind by cosmic ray anisotropy is discussed.
\end{abstract}

The 34th International Cosmic Ray Conference

30 July- 6 August, 2015

The Hague, The Netherlands

${ }^{1}$ Speaker 


\section{Introduction}

The local interplanetary parameters determine of the cosmic ray (CR) anisotropy: the IMF strength, direction and irregularity, solar wind velocity, and gradient of CR density. Accordingly to the convective-diffusion theory [1-4] vector of the CR anisotropy $\vec{A} \quad$ (the first spherical harmonic): $\quad \vec{A}=\vec{A}_{c}+\vec{A}_{d} \approx C \vec{u}+\Lambda \vec{G}$, where $\Lambda$ is the matrix of transport paths, $\vec{G}$ is a CR gradient. Convective component $\vec{A}_{c}$ is defined by local speed of the solar wind $V$ (usually directed radially from the Sun) and by Compton-Getting factor C. Any component of matrix $\Lambda$ is of the form $\lambda_{i}=k_{i} \rho$, where $\rho$ is gyroradius of particles, which is in inverse proportion to the IMF strength, and coefficients $k_{i}$ are defined by a degree of IMF irregularity.

We know which parameters determine the vector of anisotropy but these parameters are known not sufficiently. Although solar wind velocity and IMF characteristics are measured about half a century, but we may consider them as known by conventional (because the point for us and for cosmic rays means different things). For the CR point has a size of hyroradius (for the CR observable on the Earth this is 0.1 a.u. by order). Situation with other important parameters (IMF regularity and CR gradient) is much worse - they are not measured directly and there is no reliable way to determine these parameters for certain place and the moment.

For all period of solar wind measurements (even since 1957) it was succeeded to obtain characteristics of vector anisotropy of CR. It allows one to make the statistical analysis of a relation of $\mathrm{CR}$ vector anisotropy characteristics with the measured parameters of a solar wind.

Main objectives of our work is to find out how the equatorial component Axy of CR vector anisotropy depends on key parameters of the interplanetary environment; to reveal distinctions of Axy in typical situations in the quiet and disturbed solar wind; to understand, in what measure data on Axy allow to judge about conditions in the interplanetary environment.

\section{Data and Methods}

We used hourly characteristics of the CR with rigidity $10 \mathrm{GV}$ calculated by Global Survey Method [2,5] from the data of world wide NM network for the period from July 1957 to December 2013 (in total almost 500000 hours), and hourly values of the solar wind characteristics collected in OMNI Database (http://omniweb.gsfc.nasa.gov) starting from 1963.

\section{Results and Discussion}

3.1. Equatorial component of vector anisotropy We study here only equatorial component of the vector anisotropy Axy. which changes whithin a wide range: it is sometimes equal 0 , during 56 years it happened 49 times, and once (in huge FD on October 29, 2003) Axy exceeded 10\%. We will notice that Axy $=0$ doesn't mean lack of anisotropy as the North-South component of Az or higher harmonicas can significantly differ from 0 in these hours.

The average size $A x y=0.60 \%$, median $-0.53 \%$. $2 / 3$ all of Axy values vary in rather narrow range of $0.25-0.91 \%$. If take narrow range of all parameters of a solar wind near mean values, then average $\mathrm{Axy}=0.53 \%, 2 / 3$ all values are within $0.24-0.80 \%$. 
It is well known [eg. 6-7] that the direction of vector anisotropy depends on polarity of a solar dipole. But also vector size depends on it too. At approximately identical parameters of the interplanetary environment, but at various polarities the average size Axy will be various: $0.54 \%$ - at negative and $0.46 \%$ - at positive.

Axy is conceptually close to the solar diurnal anisotropy which can be seen in data of any ground level detector like a diurnal wave, and practically, Axy is the amplitude of such a wave estimated for particles of $10 \mathrm{GV}$ rigidity. Since $10 \mathrm{GV}$ is close to the effective rigidity of majority NMs (with an exception of very low latitudinal ones) then, calculated Axy are close to the amplitudes of solar diurnal wave at high latitudinal (but not subpolar) NMs. Solar diurnal anisotropy is actively studied long ago (more than half a century), distinguishing, as a rule, a daily wave from of counting rate variations by means of the harmonic analysis. The obtained thus, solar diurnal anisotropy except similarity to Axy has also essential differences from it. Main preferences of Axy: 1) it is the characteristic of angular distribution of CR intensity which isn't connected with any single detector; 2) it is hourly (not daily) characteristic. These differences give the chance of more detailed comparison of Axy with characteristics of the interplanetary environment.

3.2. Relation Axy to the solar wind velocity. Let us compare hourly values of Axy with solar wind velocity over the whole studied period -316703 hours (fig. 1).

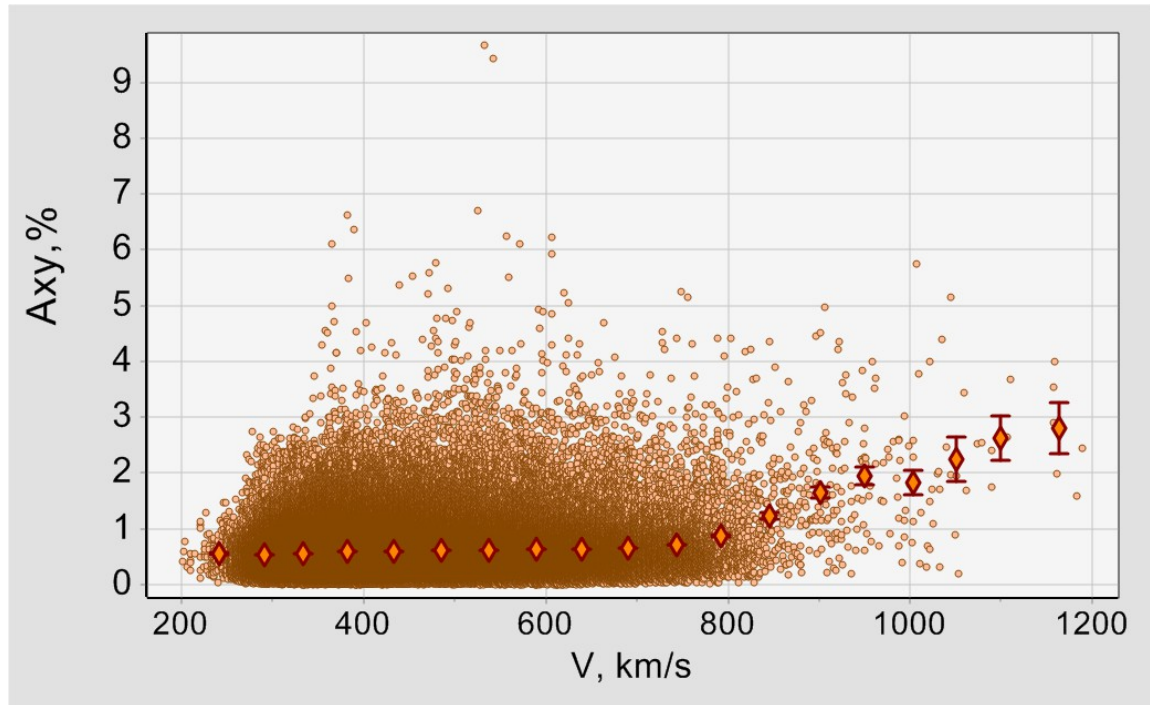

Figure 1. Axy versus to the solar wind velocity V. Circles show data for each hour, diamonds Axy averaged by equal intervals of velocity.

If the reason of the CR anisotropy was only convection we would have simple linear growth of Axy with the wind velocity. But Figure 1 shows that for the wide velocity range, up to $750 \mathrm{~km} / \mathrm{s}$, a dependence of $\mathrm{Axy}(\mathrm{V})$ is practically absent. This is a direct consequence from convective-diffusion model of anisotropy [1,2]. Dependence appears only under the highest velocities which follow the most significant interplanetary disturbances but all the same, dependence of $\operatorname{Axy}(\mathrm{V})$ remains to be weak (correlation coefficient for $\mathrm{V}>750 \mathrm{~km} / \mathrm{s}$ is only 0.37). It is natural to suppose that in these hours the magnetic field may also be strengthened, and gradient of CR density is much higher as well than usual. Thus, the increase of Axy under large velocities may be caused not only by velocity. Parameters of the interplanetary environment are closely interrelated and to distinguish an influence of some one parameter is 
difficult task. Apparently, is more perspective to consider at the same time an influence of two or several parameters. If to consider not only the size of speed of a solar wind, but also its dynamics (change of speed in an hour, $\mathrm{dV}=(\mathrm{Vk}+1-\mathrm{Vk}-1) / 2$ where $\mathrm{k}$ is a number of hour), we will see stronger dependence and more interesting picture (fig. 2).

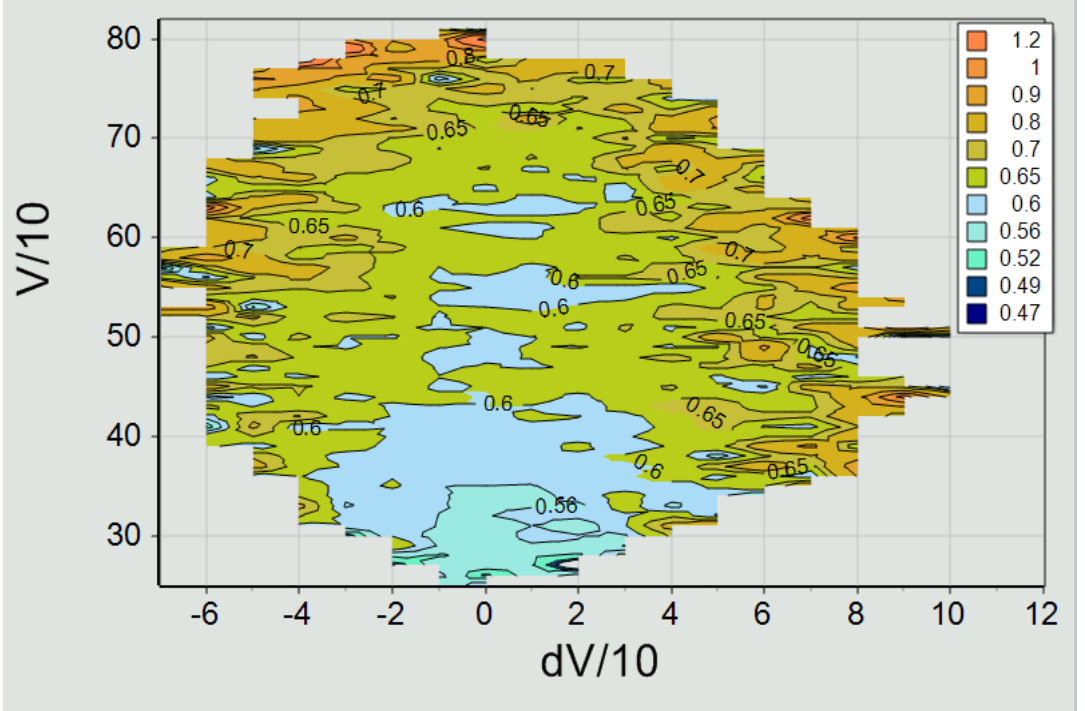

Figure 2. Relation Axy to the solar wind velocity V and its changes $\mathrm{dV}$ for one hour. Counter isolines correspond to equal values of Axy.

Follows from fig. 2 that dependence Axy on changes of speed of dV (more precisely, on $\operatorname{abs}(\mathrm{dV}))$ is stronger, than dependence on the speed. It thus that there is no straight (expected from the theory) anisotropy relation to $\mathrm{dV}$. In this case changes of a solar wind speed act as the indicator of a disturbance of the interplanetary environment. Growth of speed is usually connected with interaction of streams of a wind, thus the probability of strengthening of IMF and appearance of considerable gradients of CR density is great in these places. Considerable and fast speed decrease, as the rule, is also observed in disturbed solar wind. Fast change of speed of a solar wind is a sign of the interplanetary disturbance and, most likely, dependence of $\operatorname{Axy}(\mathrm{dV})$ is explained by it.

3.3. Relation of Axy to the IMF intensity. Such a relation has to be, but it is very difficult to receive it in pure form, it is even more difficult, than to search for correlation between Axy and a speed of a solar wind. If the high-speed and not disturbed solar wind can be imagined, the high IMF strength is always connected with interplanetary disturbances. Fig. 3 shows that dependence $\operatorname{Axy}(B)$ is weak $(C C=0.26)$ though it it is obviously stronger, than of $\operatorname{Axy}(V)$ relation. Increase of Axy together with B is visible up to very big values of IMF intensity. And only at $\mathrm{B} \approx 35-40 \mathrm{nT}$ it stops.

In power representation of $A x y(B)$ it looks like $A x y \propto B^{0.28}$. The magnetic field generally interferes of charged particle transfer and reduces anisotropy. At a gradient $\mathrm{g}$ of $\mathrm{CR}$ density Axy $\propto \rho g \propto g / B$. However the gradient, isn't constant, and correlates with intensity of a magnetic field. The stronger field, the more probable a big gradient is. From our result it follows that dependence of a CR gradient on B is stronger than the linear. 


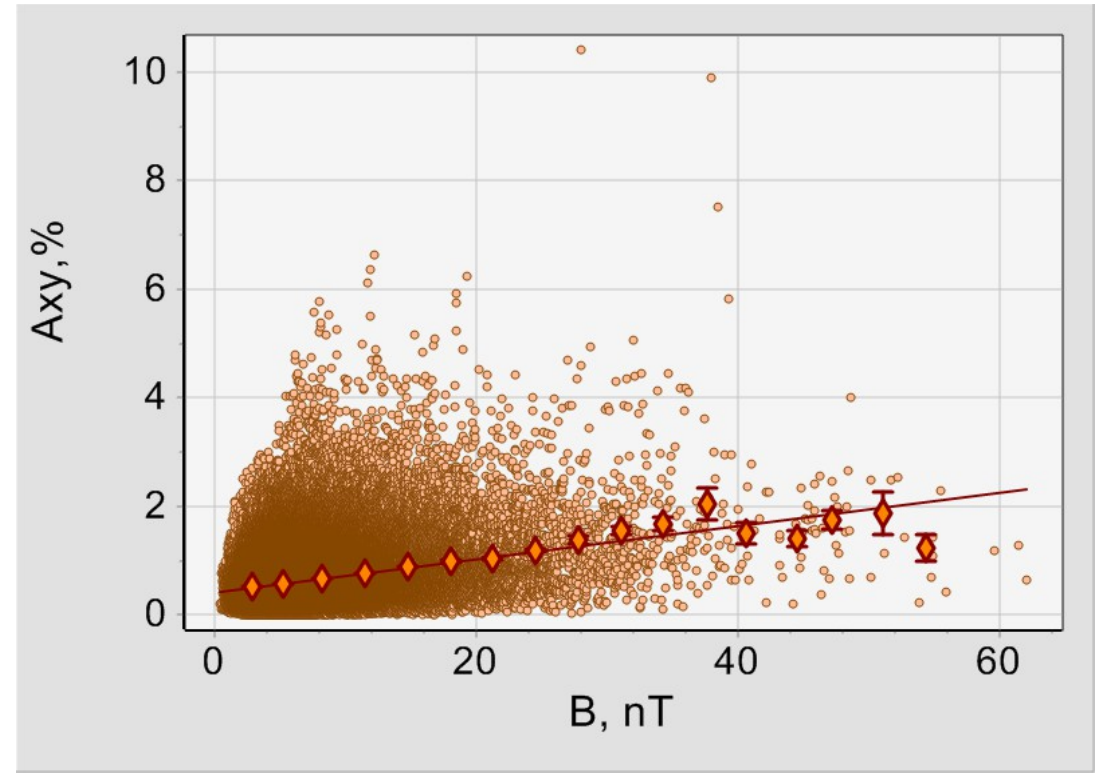

Figure 3. Dependence of Axy on the IMF intensity B. Points are hourly values of Axy. Diamonds mean Axy averaged within the equal intervals of B.

We have to remember that together with IMF intensity other parameters of interplanetary environment are also changing (for example, solar wind velocity). Fig. 4 demonstrates the changes of Axy under $\mathrm{V}$ and $\mathrm{B}$ variations.

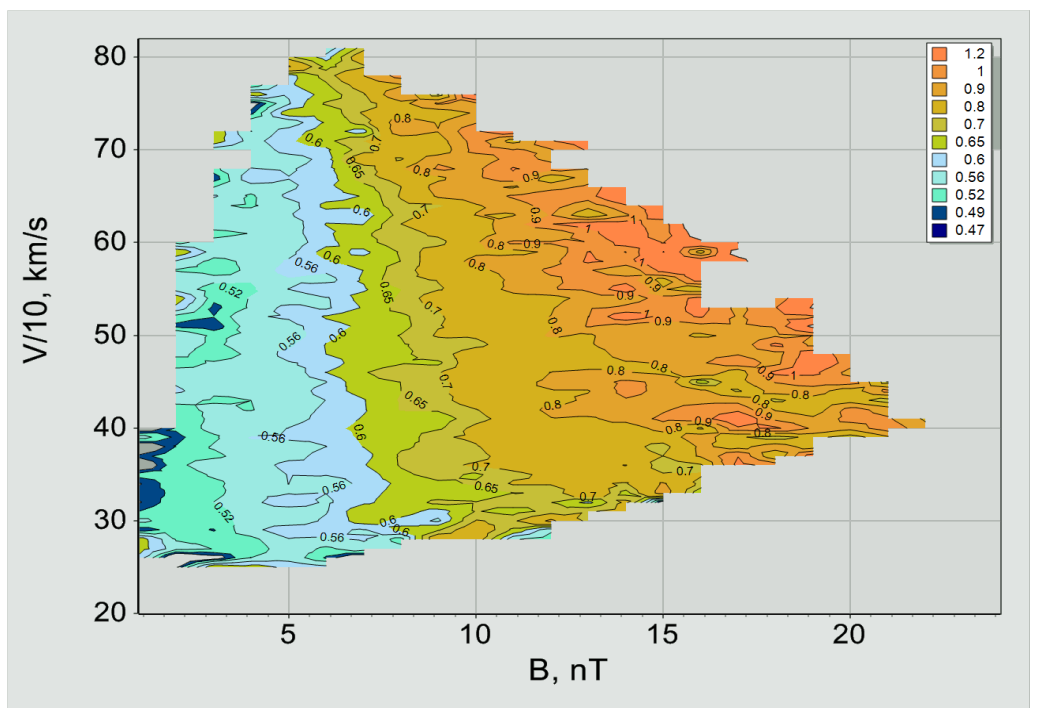

Figure 4. Connection of Axy with the solar wind velocity V and IMF intensity B. Different colours mark different ranges of rigidities (right scale).

At $\mathrm{B}=4 \div 6 \mathrm{nT}$ that is close to average sizes, Axy almost doesn't change with changes of SW speed as it seen also in fig. 1-2. At the strengthened magnetic field the increased sizes of Axy are observed at all values of speed of a solar wind and Axy grows with increase in speed. At low speeds and the weaker field the size of Axy is lowered though at the lowest values of B the signs of some increase in Axy exist. 
3.4. Connection with $C R$ gradient. Gradient of $C R$ density is the main reason of the $C R$ anisotropy. Unfortunately, we don't know the CR gradient in a definite moment in a fixed point. However under certain circumstances changes of the CR density can give the information on CR gradient. In our work we use density variations (A0) of the CR with rigidity $10 \mathrm{GV}$, obtained by global survey method (GSM) [5] together with characteristics of CR anisotropy. We consider that at hour $\mathrm{k}$ the changes of $\mathrm{CR}$ density variation is $\mathrm{dA} 0=\left(\mathrm{A} 0_{\mathrm{k}+1}-\mathrm{A} 0_{\mathrm{k}-1}\right) / 2$. In some situations dA0 can give an information on some components of a CR density gradient: about a radial component when passing ICME and about an azimuthal component in high-speed streams from the coronal holes.



Figure.5. Relation of the Axy vector to the changes of CR density (dA0). Regression lines are obtained separately for positive and negative dA0.

In fig. 5 one can see that there is some Axy dependence on an absolute value of dA0. For negative dA0 this connection looks more remarkable (correlation coefficient is 0.31 ), but this results is possible due the fact that there is much more significant negative $\mathrm{dA} 0$, than positive. Certainly, changes of CR density happen not in itself, and together with changes in a solar wind, with responding to them by a complicate way. One of the most essential characteristics to changes of CR density is IMF strength. In fig. 6 joint influence of these characteristics (B and $\mathrm{dA} 0$ ) on vector anisotropy of CR (Axy) is shown.

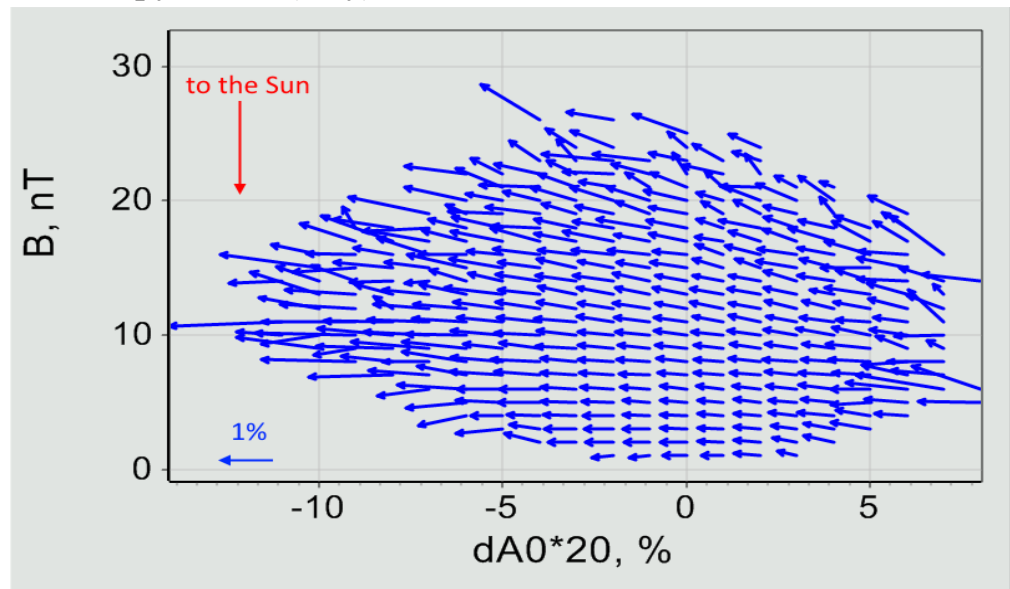

Figure 6. Connection of $\overrightarrow{A x y}$ with the changes of CR density dA0 and IMF intensity B. 
To derive Figure 6 the whole area of changes was divided on the cells of the size $1 \mathrm{nT} * 0.05 \% / \mathrm{hrs}$, and vector CR anisotropy was averaged in each cell. For small dA0 and normal IMF intensity a big number of hours was averaged in each cell (up to 2000). Under big changes of CR density and stronger IMF the number of hours was significantly less. The results are plotted only for the cells where a number of hours was $\geq 10$. One can see that points with big value abs(dA0) under lower or normal IMF intensity (B) are absent in this picture. In Figure 6 not only magnitude of $\overrightarrow{A x y}$ but also its direction is shown. Fig. 6 shows that at little changes of the CR density and at the normal or a slightly strengthened IMF there is a zone of stable anisotropy where length and the direction of a vector $\overrightarrow{A x y}$ almost don't depend on dA0 and B. But if one of these values (or both) significantly differs from norm, the size Axy increases, and the direction of a vector $\overrightarrow{A x y}$ becomes unstable. In general, the direction to the west remains, but also deviations from it become considerable, and vectors from the Sun are observed more often than to the Sun. Such anisotropy isn't surely connected with observed changes of CR density, but, nevertheless, is defined by the CR gradient (first of all, by radial) which exists constantly, slowly changing and not revealing itself in variations of CR density.

3.5. What can the CR anisotropy tell about? Of course, we cannot receive the exact information on a solar wind from CR anisotropy, as no unambiguous compliance between characteristics of anisotropy and parameters of the interplanetary environment exists. Nevertheless, the useful information from the CR anisotropy data can be drawn.

We will review concrete examples. Let Axy in a certain period be in $0.004 \div 0.006$ limit. We will look, what probability is that during this period the solar wind will be quiet (conditionally, with IMF B intensity $<7 \mathrm{nT}$ and the speed $\mathrm{V}<500 \mathrm{~km} / \mathrm{s}$, Q-wind) or disturbed (conditionally, with IMF B >15 nT and the speed V>600 km/s, D-wind). From 293429 hours with measurements of $\mathrm{V}$ and $\mathrm{B}$, more than a half $(54.9 \%)$ belongs to Q-wind, and only 1103 hours $(1.1 \%)$ to $\mathrm{D}$-wind. If we know that $\mathrm{Axy}=0.004 \div 0.006$, probability of Q-wind increases to $56.9 \%$, and the probability of D-wind decreases almost by order to $0.13 \%$.

Another example. Let Axy is abnormally large $(>0.02)$. The number of such hours coinciding with measurements of a solar wind was only 2545 with the share of Q-wind only 552 hours $(21.7 \%)$, i.e. it was much less, than at normal values of Axy. But 213 hours (8.4\%) fall on D-wind and the probability of disturbed wind in comparison with a case of normal anisotropy increases by 64 times.

Thus, the accounting of only one characteristic of CR - the size of Axy - can change radically our ideas on possibility of essential solar wind disturbances during a certain period. And if to add other available characteristic - change of CR density (dA0), informational content of $\mathrm{CR}$ will increase. Again we will review 2 examples: quiet ( $\mathrm{Axy}=0.004 \div 0.006$, $\operatorname{abs}(\mathrm{dA} 0)<0.0005)$ and disturbed $(\mathrm{Axy}>0.02, \mathrm{dA} 0<-0.005) \mathrm{CR}$ conditions. The defined thus, quiet condition of CR was observed 42945 hours. From them 26325 hours (61.3\%) corresponded to Q-wind and only $16(0.037 \%)$ - E-wind. We will consider the disturbed condition of CR for comparison. Among the 651 hours only 45 hours (6.9\%) corresponded to Qwind and $123(18.9 \%)$ to E-wind. From this it follows that the probability of disturbed condition of a solar wind changes depending on a condition of CR by 507 times. No doubts that other CR characteristics (N-S anisotropy, direction of the vector anisotropy, the second harmonic, etc.) can increase informational content of $\mathrm{CR}$ variations. 


\section{Main Conclusions}

On the big volume of hourly experimental data covering almost entire period of solar wind measurements we studied the relation of the equatorial component of vector CR anisotropy with interplanetary characteristics: with the solar wind speed and its changes, with an interplanetary magnetic field intensity, with changes of CR density.

Any measured or easily counted parameter of the interplanetary environment doesn't show close correlation with the size of CR vector anisotropy. However an increase of vector anisotropy follows to a combinations of the parameters of the environment manifested its disturbances. Most obviously increase of anisotropy is promoted by considerable changes of CR density and speed of a solar wind, and also strengthening of the IMF.

The size of vector anisotropy of CR almost doesn't depend on the speed of a solar wind as it was predicted by convective-diffusive model of anisotropy.

Weak positive correlation of Axy with IMF intensity B, apparently, testifies to stronger dependence on B for radial gradient of CR density.

Statistical relations of parameters of anisotropy to various conditions of the interplanetary environment give the information on degree of environment disturbance. The accounting of the size Axy (separately or together with variations of CR density) can change probability of the quiet or disturbed solar wind in tens and hundreds times.

\section{Acknowledgements}

The authors are thankful to collaborators of the world wide network of CR stations providing data of continue monitoring of neutron component: http://cr0.izmiran.ru/ThankYou/. We acknowledge also the NMDB database (www.nmdb.eu), founded under the European Union's FP7 program (contract no. 213007) for providing data from NM worldwide network.

\section{References}

[1] Krymsky G. The diffusion mechanism of the diurnal variation of cosmic rays. Geomagnetism and Aeronomy 4 (1964) 977-985 (in russian)

[2] Krymsky G. Modulation of Cosmic Rays in Inteplanetary Space. USSR Academy of Sciences. Moscow (1969)

[3] Krymsky G. et al. Cosmic rays and solar wind, Nauka, Novosibirsk (1981) p 224 (in russian).

[4] Forman M.A., Gleeson L.J. Cosmic-ray streaming and anisotropies. Astrophysics and Space Science. 32 (1975) 77-94

[5] Belov A., Baisultanova L., Eroshenko E., Mavromichalaki H., Yanke V., Pchelkin V., Plainaki C., Mariatos G. Magnetospheric effects in cosmic rays during the unique magnetic storm on November 2003. J. Geophys. Res. 110 (2005) doi:10.1029/2005JA011067

[6] Duggal S., Forbush S., Pomerantz M. Variations of the diurnal anisotropy with periods of one and two solar cycles. Proc. 11-th ICRC (1970) 55-59

[7] Forbush S., Beach L. Cosmic-ray diurnal anisotropy and the Sun's polar magnetic field. Proc. 14-th ICRC (1975) 1204-1208 